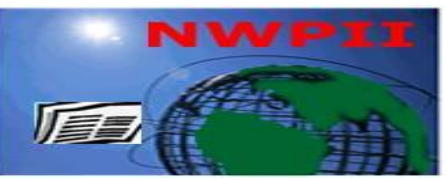

American Journal of Biomedical Sciences

ISSN: 1937-9080

nwpii.com/ajbms

\title{
Drug Delivery Stimulation by Alternating Current Using Methylene Blue Dye Absorption by Pig's Skin as a Model
}

\author{
Lobna A. Elnahas ${ }^{1}$, Mohamed A. Elblbesy ${ }^{1 *}$,Youssef S Youssef ${ }^{1}$, Thanaa E Shalaby ${ }^{1}$, \\ Mona A Yehia ${ }^{2}$
}

\author{
${ }^{1}$ Bio-Medical Physics Department, Medical Research institute, Alexandria University, Egypt. \\ ${ }^{2}$ Histochemistry\& Cell Biology Department, Medical Research institute, Alexandria University, Egypt. \\ *Corresponding Author: \\ Mohamed A. Elblbesy, Ph.D. \\ Bio-Medical Physics Department \\ Medical Research institute \\ Alexandria University \\ Alexandria, Egypt \\ Email:mimizizo@yahoo.com
}

Received:16 July2012; | Revised:18 September2012; | Accepted: 4 October 2012

\begin{abstract}
Pig skin has been studied as administration site of drug for its systemic effects, since systemic therapeutic agents can be delivered for long time with a controlled ratio, escaping from the first pass effect by liver through the transdermal delivery, which can decrease the drug dose. The low permeability of drug molecules through stratum corneum(SC) has been the limiting factor for developing transdermal delivery system of therapeutic agents. The aim of the present work is to study the enhancement of drug absorption by the skin using stimulating alternating current (AC). In our study stimulated voltage over range $6.5 \mathrm{~V}$ to $200 \mathrm{~V}$ had been used to enhancement the absorption of methylene blue (MB) with pig's skin. Our results showed that the highest MB concentration in tissue was $(0.239 \pm 0.011) \mathrm{mg} / \mathrm{g}$ and lowest ohmic resistances $(\mathrm{k} \Omega / \mathrm{cm})$ $(3.15 \pm 0.223)$ for skin were obtained after skin stimulation with $12.5 \mathrm{~V}$; these results had been confirmed using histochemistry study. Dielectric properties of skin in the range of $10 \mathrm{~Hz}-100 \mathrm{KHz}$ revealed change in the dielectric dispersion curves immediately after skin stimulation.
\end{abstract}

Keywords: Alternating current -Drug Delivery - Methylene Blue - Pig skin.

\section{Introduction}

Topical drug administration has potential advantages over oral, injection, or intravenous drug delivery. These advantages include convenience, noninvasiveness, minimal trauma induction, and avoidance of "first-pass" degradation or metabolism in the gastrointestinal tract (GIT) or liver. Tightly localized administration is possible, and systemic delivery can be achieved through absorption by the dermal blood supply. The main barrier to cutaneous or transcutaneous drug delivery is the 
impermeability of thestratum corneum (SC), which is outermost layer of the skin, constitutes a formidable barrier both to the loss of tissue water and to entry of xenobiotics into the skin. If the integrity of SC is disrupted, the barrier to molecular transit may be greatly reduced and facilitate the transdermal drug delivery(Guy R1996,Delgado et al.2001;Singhet al. 2002;Kalia, Yet al. 2004).

Transdermal administration of drug is assuming an important place in modern drug therapy. Transdermal administration can be passive or facilitated. In passive administration, the non-ionized drug transverses the skin through the lipophilic SC(Kassan D et al. 1996), the lipidprotein matrix of SC not only restricts the passive diffusion of lipophilic molecules but also severely limits the transport of hydrophilic compounds across the membrane (Narasimha M, et al.2007). This matrix makes the skin a semipermeable membrane, allows only a small amount of any drug molecules, which are small in size and rather lipophilic in nature, to passively penetrate the skin(Kassan D et al. 1996). Ionized drugs do not easily penetrate this barrier and are not suitable for routine transdermal delivery unless an external source of energy is provided to drive the drug across the skin(Green Pet al.1993). Facilitated diffusion can be utilized by chemical enhancers such as low molecular alcohols (Bhatia K, and Singh J1999), aliphatic esters, and various vehicle formulations(Williams $\mathrm{A}$, and Barry B2004;Asbill C et al. 2000). Other methods rely on physical techniques such as iontophoresis(Chien Y, and Banga A1989), electroporation (Prausnitz M et al. 1993), and phonophoresis(Mitragori S et al. 1995).

Iontophoresis is a method of transdermal local drug delivery using electrical current(Burnette $\mathrm{R}$ et al. 1989)iontophoresis increases the penetration of electrically charged drugs into surface tissues. Electrical energy assists the movement of ions across the SC according to the basic electrical principle like charges repel each other and opposite charges attract each other(Honggang $S$ et al. 2002). The drug is applied under an electrode of the same charge as the drug (delivery electrode) and a return electrode opposite in charge to the drug which is placed at a minimal distance from the other electrode at a neutral site on the body surface (dispersive electrode)(Wiliam E et al. 2005).

The applied electric current must be below the level of patient's pain threshold. The two classically considered prerequisites for iontophoretic treatment are that the drug must be charged (or modified to carry a charge) and the distance process must be at or near a body surface(Peter $\mathrm{M}$ et al. 2003).

Iontophoresis enhances transdermal drug delivery by three mechanisms: (a) ion-electric field interaction provides an additional force that drives ions through the skin, (b) the flow of electric current increases the permeability of the skin, and (c) electro-osmosis produces bulk motion of solvent that carries ions or neutral species with the solvent stream(Zhu $\mathrm{H}$ et al. 2002).

Iontophoresis provides a controlled delivery rate of ionized drugs through variations of current density, pulsed voltage, drug concentration and ionic strength. It eliminates the gastrointestinal incompatibility, erratic absorption, and first pass metabolism. It reduces side effects and avoids the risks of infection, inflammation, and fibrosis associated with continuous injection or infusion. It enhances patient compliance with a convenient and noninvasive therapeutic regimen, and decreased dosing frequency. It improves efficacy by continuous release and decreases the total dose and dosing frequency(Reena $\mathrm{R}$ and Srinivas C2005).

Iontophoresis has been proposed for numerous uses, including delivering local anesthetic before skin puncture or painful dermal procedures, treatment of excessive sweating of the hands and feet, local drug delivery for agents such as nonsteroidal anti-inflammatory drugs or corticosteroids for musculoskeletal inflammatory disorders; and others(Pradas R et al. 2006; Khoo $P$ et al. 2006).

Methylene blue (MB) dye is used in some researches because its color allows visual observation of its location; its absorbance and fluorescence properties permit spectroscopic detection. Its cationic nature can be used as a 
model for a number of photosensitizes and chemicals with similar characters (in which the methyl group is replaced by longer chain)(Kirste $\mathbf{J}$ et al. 2002)and has similar physicochemical properties to lidocaine (anesthetic drug)(Flucker Cet al.2000).

Pig skin was selected for the in vitro experiments, because it has histological properties comparable to human skin with similarities in epidermal thickness and composition, pelage density, dermal structure, lipid content, and general morphology(MonteiroRiviere N 1986). The net charge on the skin is zero when the degree of permselectivity (DP) equals unity, corresponding to the IP of the membrane $(\sim 4.4$ for pig skin and $\sim 4.8$ for human skin). The consistent IPs observed for human and pig demonstrate that porcine skin is an appropriate model for iontophoresis studies (Diego $\mathrm{M}$ et al. 2001). Furthermore, in a number of permeation studies, pig skin showed to be a good model for human skin permeability (Chang S, and Riviere J 1991; Woolfson A et al.1992)

The aim of the present work is to study the effect of stimulating alternating current (AC) on the absorption of MB by pig's skin. This to evaluate how can stimulate voltage accelerate the process of drug delivery.

\section{Materials and methods}

\subsection{Skin sample}

Fresh pieces of abdominal skin from domestic pigs were obtained from a local abattoir with dimensions $\approx 50 \times 60 \mathrm{~cm}^{2}$. Samples, were stored on ice, and used within 48 hours. The skin was shaved by electric clippers to remove all obvious hairs. The skin was then cut into square pieces each one has the erea15×15 $\mathrm{cm}^{2}$. Before use the skin was washed three times with normal saline then with distilled water. This method was repeated for every piece of skin obtained.

\subsection{Electrodes}

Two electrodes of diameter $\Phi=9 \mathrm{~mm}$ made of $\mathrm{Ag} / \mathrm{AgCl}$ (Leonhard Lang Gmb H "UK" FS-521), which they already used in electrocardiogram purposes and their original function are to transmit the electrical signals across the skin were used and fixed to the skin by adhesive tape.

\subsection{Silver-silver chloride ring}

Two rings manufactured from $\mathrm{Ag} / \mathrm{AgCl}$, its diameter $\Phi=9 \mathrm{~mm}$ as electrode diameter, and its height is $5 \mathrm{~mm}$. The rings were put between the electrodes and the skin. They acted as vehicle to the drug

(wetted cotton piece with $\mathrm{MB}$ put in the ring), also the silver ring used to determine the size of punch of skin sample $\Phi=9$ $\mathrm{mm}$.

Seven main pig skin groups used in the work classified as the following:

- G I: 40 samples were stimulated by pulsing half sine shape waves; the pulse height used in this group was $6.5 \mathrm{~V}$, and its duration 5 seconds.

- G II: 40 samples were stimulated by pulsing half sine shape waves; the pulse height used in this group was $12.5 \mathrm{~V}$, and its duration $5 \mathrm{~s}$.

- G III: 40 samples were stimulated by pulsing half sine shapes waves; the pulse height used in this group was $25 \mathrm{~V}$, and its duration $5 \mathrm{~s}$.

- G IV: 40 samples were stimulated by pulsing half sine shape waves; the pulse height used in this group was $50 \mathrm{~V}$, and its duration $5 \mathrm{~s}$.

- GV: 40 samples were stimulated by pulsing half sine shape waves; the pulse height used in this group was $100 \mathrm{~V}$, and its duration $5 \mathrm{~s}$.

- GVI: 40 samples were stimulated by pulsing half sine shape waves; the pulse height used in this group was $200 \mathrm{~V}$, and its duration $5 \mathrm{~s}$.

- $\mathrm{G}^{\circ}$ : 40 samples weren't stimulated by pulsing half sine waves and were used as control.

\subsection{Methylene blue dye preparation}

$1.122 \mathrm{gm}$ from the MB crystal was taken and dissolved in $100 \mathrm{ml}$ of distilled water to obtain 0.03 M.A specified constant volume of this concentration was used along this study $(200 \mu \mathrm{l})$ and it was determined in the preliminary 
experiments.But the MB which diffused to the skin was determined in basic medium (ammonium hydroxide) $\left(\mathrm{NH}_{4} \mathrm{OH}\right)$, so the physical and chemical parameters of the MB was determined in basic medium and compared with the characteristics in the aqueous solution.

\subsection{Stimulating circuit}

Stimulation of the skin was achieved by half-sine waves with different peaks voltages. It was applied to examine the effect of this pulsing stimulus on the absorption of MB.The circuit that shown in figure 1 was attached to the electrodes fitted with $\mathrm{Ag} / \mathrm{AgCl}$ rings to the skin. The stimulating currents were changed to give $(6.5 \mathrm{v}$, $12.5 \mathrm{v}, 25 \mathrm{v}, 50 \mathrm{v}, 100 \mathrm{v}$, and $200 \mathrm{v}$ ), the duration of this stimulation was 5 seconds. The ohmic resistance was measured within 9 seconds after terminating the stimulus.

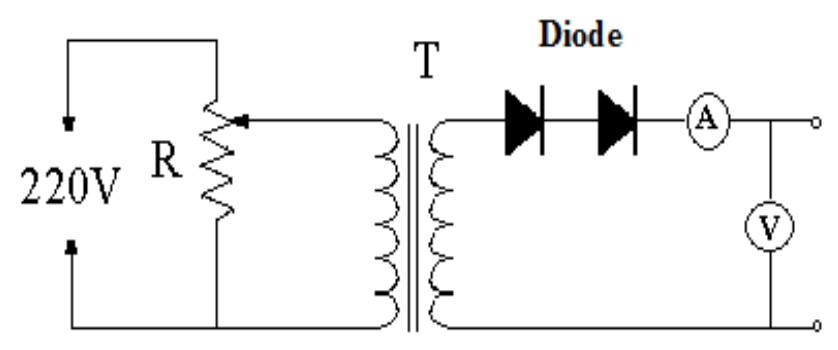

Figure 1.Pulsing sine wave producing circuit

\subsection{Experiment set-up}

Skin was put dermal side down, on ice covered with cling film. Each skin section was used once. Two $\mathrm{Ag} / \mathrm{AgCl}$ rings were placed on the skin surface $(8 \mathrm{~cm}$ apart), then a piece of cotton containing $200 \mu \mathrm{l}$ of the prepared MB solution was put in cavity of one ring which was used as positive electrodes (anode). The two electrodes were fixed to the skin above the two $\mathrm{Ag} / \mathrm{AgCl}$ rings by adhesive tape; a slight pressure was applied evenly to the electrodes to ensure a good contact to the skin.

\subsection{Absorbance spectra measurements}

Punched samples of $9 \mathrm{~mm}$ diameter were taken underneath the positive electrode (anode), its $\mathrm{WT} \approx(0.3 \pm 0.1 \mathrm{gm})$. Each sample was rinsed in water to remove the superficial dye then it was dissolved overnight in $1 \mathrm{ml} \mathrm{NH}_{4} \mathrm{OH}$. The Solution was centrifuged, and absorbance spectra of the supernatant fractions were recorded spectrophotometricaly $(\lambda=670 \mathrm{~nm})$, to monitor MB level. After measuring the absorbance of $\mathrm{MB}$, the concentration of MB in (mole/liter) was calculated according the following equation:

$$
M B \text { conc. }=\frac{\text { Analyt }- \text { Blank }}{\begin{array}{l}
\text { Standard }- \text { Blank } \\
\times \text { conc. standard .......... }
\end{array}}
$$

\subsection{Histological method}

Preparation of frozen section:The skin punch was used to prepare a frozen section. It was placed in a holder in a refrigerated chamber (-60 degrees). Then the samples were cut into small sections $(10 \mu \mathrm{m})$.

Procedure: The section was fixed at a $10 \%$ formaldhyde, washed in dis. $\mathrm{H}_{2} \mathrm{O}$. The nuclear fast red was used as a counter stain, then left the slide to dry and mounted by glycerine-gel and covered by cover slip to be observed under light microscope, and then a photograph was taken with digital compact camera.

Image analysis: The image analysis of the depth of invasion (penetration depth) of color was measured by soft imaging system(Pearse A 1972)

\subsection{Dielectric properties of the skin}

Dielectric properties of the skin (the relative permittivity, the dielectric loss factor, and the conductivity) were determined after applying the stimulating voltage immediately. The measured capacitance "C", and resistance "R" of pig skin were measured over frequency range from $12 \mathrm{~Hz}$ to $100 \mathrm{KHz}$, with LCR meter. Skin tissue which taken under the positive electrodes was placed in glass measuring cell between two circular disc electrodes of silver (figure. 2). The skin was first stimulated by stimulating circuit using $(6.5 \mathrm{v}$, $12.5 \mathrm{v}, 25 \mathrm{v}, 50 \mathrm{v}, 100 \mathrm{v}$, and $200 \mathrm{v}$ ), for $5 \mathrm{~s}$ then the dielectric properties of the skin were measured by LCR- Bridge after fixed time (90s).Control was done by measurement the dielectric property directly without any stimulation. This was done at room temperature which was maintained constant at $25^{\circ} \mathrm{C}$. 


\section{Results}

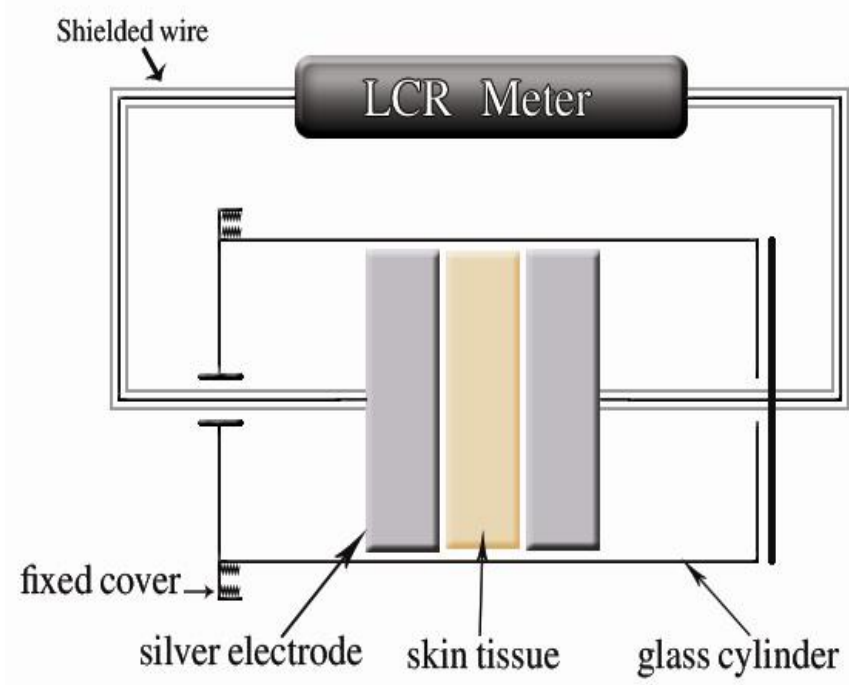

Figure 2.Diagram of the dielectric measuring cell

The relative permittivity $(\varepsilon)$, the dielectric loss factor $\left(\varepsilon^{\prime \prime}\right)$, and the conductivity $(\sigma)$ were calculated according the following equations.

$$
\begin{aligned}
& \varepsilon^{\prime}=\frac{\text { Air capacitance }}{\text { Tisssue capacitance }} \ldots \ldots \ldots \ldots \ldots .2 \\
& \text { Air capacitance }=\frac{\varepsilon_{o} A}{d} \ldots \ldots \ldots \ldots \ldots \ldots 3 \\
& \varepsilon^{\prime \prime}=\frac{\varepsilon^{\prime}}{2 \pi f R C} \ldots \ldots \ldots \ldots \ldots \ldots .4 \\
& \quad \sigma=\frac{d}{R A} \ldots \ldots \ldots \ldots \ldots \ldots 5
\end{aligned}
$$

Where:

$\varepsilon_{\mathrm{o}}$ is the vacuum permittivity which equals 8.85 $\times 10^{-12}$ farad $/$ meter.

$\mathrm{A}$ is the electrode surface area in meter square. $\mathrm{d}$ is the distance between the two electrodes in meter.

$\pi=3.14$

$\mathrm{f}$ is the frequency in $\mathrm{Hz}$.

$\mathrm{R}$ is the resistance in $\Omega$.

$\mathrm{C}$ is the capacitance in Farad.

oinsimen/meter.

\subsection{Statistical analysis}

All the parameters measured were represented as the mean $\pm \mathrm{Sd}$. Correlations between different parameters were studied using Excel Microsoft 2007.
Electrical studies in the present work showed the effect of stimulating voltage from 0 $200 \mathrm{~V}$ on the MB concentration (in $\mathrm{mg} / \mathrm{g}$ tissue). The concentration percentage reached its highest value at 12.5 volts and began to decrease again (figure 3). Strong correlation between the concentration of $\mathrm{MB}$ and stimulating voltage was found $\left(\mathrm{R}^{2}=0.73\right)$.

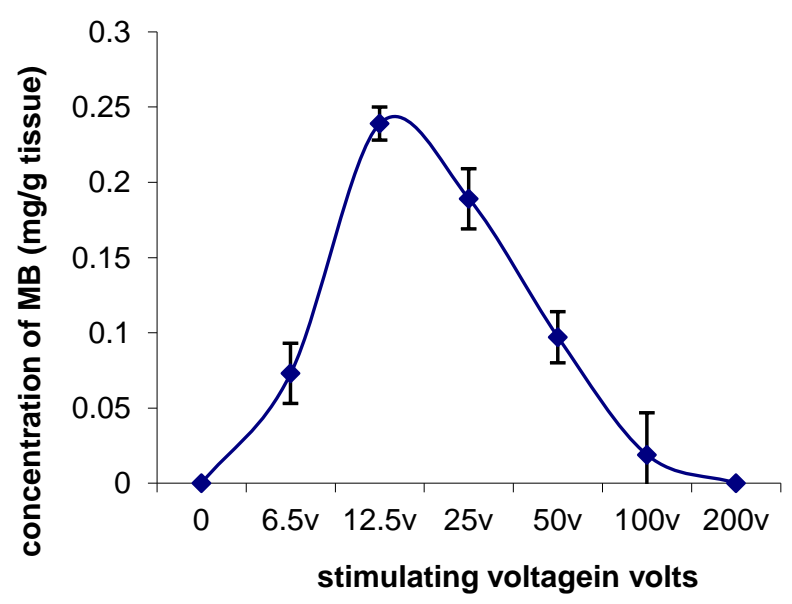

a- Dependence of the absorption of $\mathrm{MB}(\mathrm{mg} / \mathrm{g})$ on the stimulus voltage value

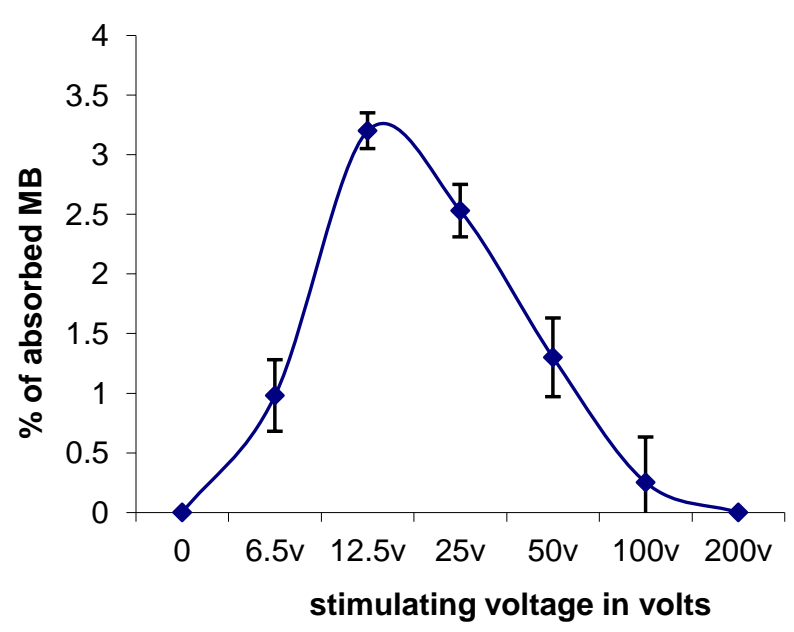

b- The percentage of the absorbed $\mathrm{MB}$ as the stimulating voltage varies

Figure 3. Effect of stimulus voltage value on the absorption of MB through pig skin. 


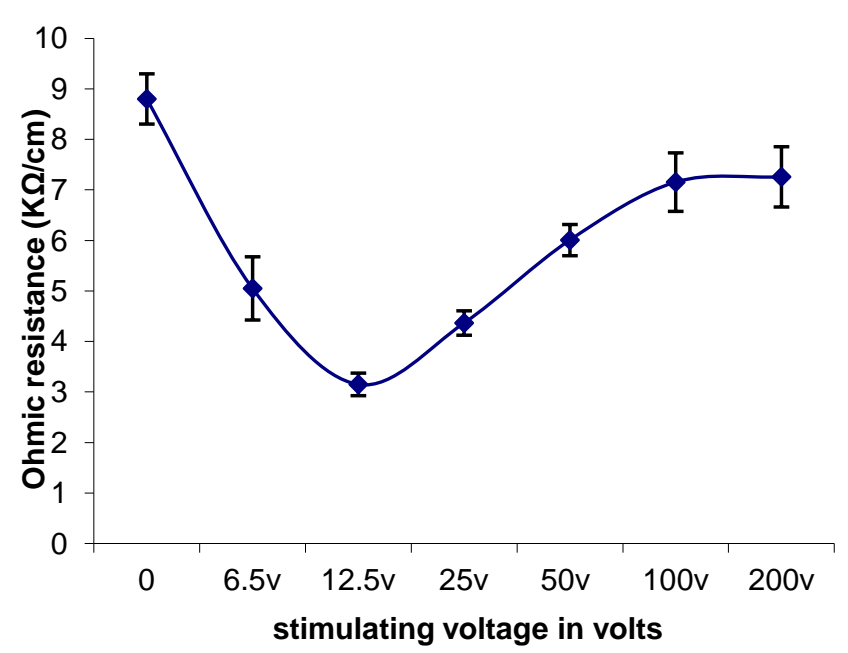

Figure 4. Relationship between the value of the stimulating voltage and the ohmic resistance of the skin in $(\mathrm{K} \Omega / \mathrm{cm})$.

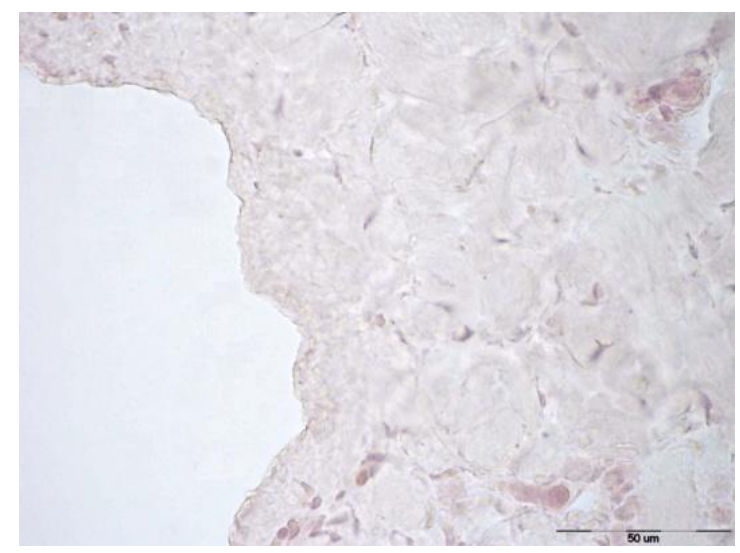

Figure 5. Frozen section micrograph of pig skin stained by nuclear fast red showing the anatomical structure of skin layers $(X=400)$.

The ohmic resistance of the skin was decreased as the stimulating voltage increased. It reached the lowest values at $12.5 \mathrm{~V}$. From Figure 4. It's clear that the ohmic resistance began to increase for the stimulating voltages higher than $12.5 \mathrm{~V}$.

In comparison between the control skin sample and stimulating samples with AC, histological study showed a clear difference between them in absorption of MB.Figure 6 shows the light micrographs of the correlation of MB penetration depth to the anatomical structure of the skin after stimulation via $\mathrm{AC}$, the dye enters the SC and its penetration depends on the exposure dose particularly field application time and voltage. The penetration of $\mathrm{MB}$ increases following electropration and reaches its highest value at exposure dose of $12.5 \mathrm{~V}$ and its lower value at $200 \mathrm{~V}$.

Figure.7 shows the relative permittivity of the skin after each stimulating voltage as a function of frequency; the results indicate that the relative permittivity at 100 and $200 \mathrm{~V}$ are lower than control and the other stimulating voltages are higher than it and reaches the highest at 12.5 V. Fig.8 illustrates the dielectric loss results and similar results to relative permittivity are obtained. Figure. 9 shows the conductivity results which match with relative permittivity and dielectric loss results.

\section{Discussion}

The major flux enhancing mechanisms of AC transdermal iontophoretic transport include electrophoresis (direct electric field effect), electroosmosis (convective solvent flow), and electric field-induced pore formation (electropermeabilization) (Qingfang $\mathrm{X}$ et al., 2009).

The ability of AC to reduce and control epidermal membrane electrical resistance also makes this a useful method to improve ionexchange membrane enhanced iontophoresis. A previous study (Xu et al.,2009) has shown that the incorporation of an ion exchange membrane having a charge opposite to that of the drug ion in an iontophoresis system can enhance transdermal iontophoretic transport (i.e., permeant transference number) by approximately 2 to 4 times when the electrical resistance of epidermal membrane was lowered to and maintained at 0.5 $\mathrm{k} \Omega$ with $\mathrm{AC}$.

$\mathrm{AC}$ is more effective in sustaining a constant state of pore induction inepidermal membrane, and constant skin resistance AC iontophoresis has been shown to have less transdermal iontophoretic flux variability for neutral permeants than conventional DC (Zhu et al., 2002). (Shibaji et al., 2001) has concluded that AC enhances membrane transport by 
increasing the electromobility of the ions as a result of the decrease in the hydrated ion radii under the AC electric fields.

The increase in $\mathrm{MB}$ concentration results from the fact that different aspects of the electroporation, transport phenomenon are being

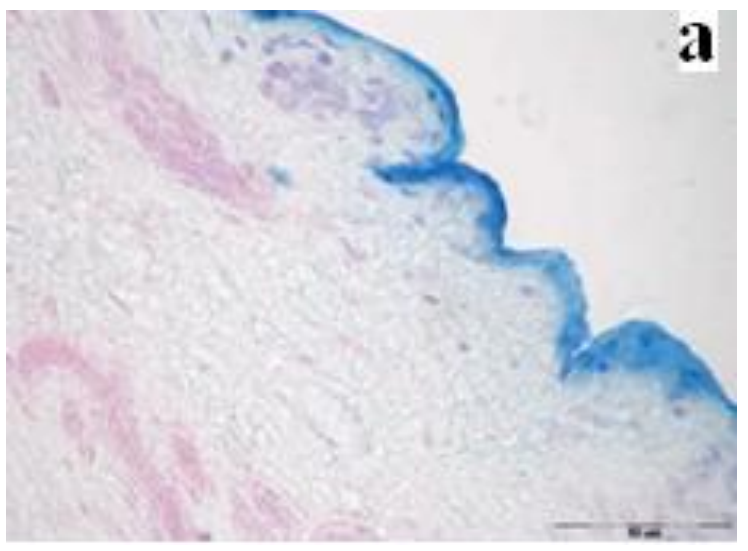

a: pig skin stimulated with 6.5 volts

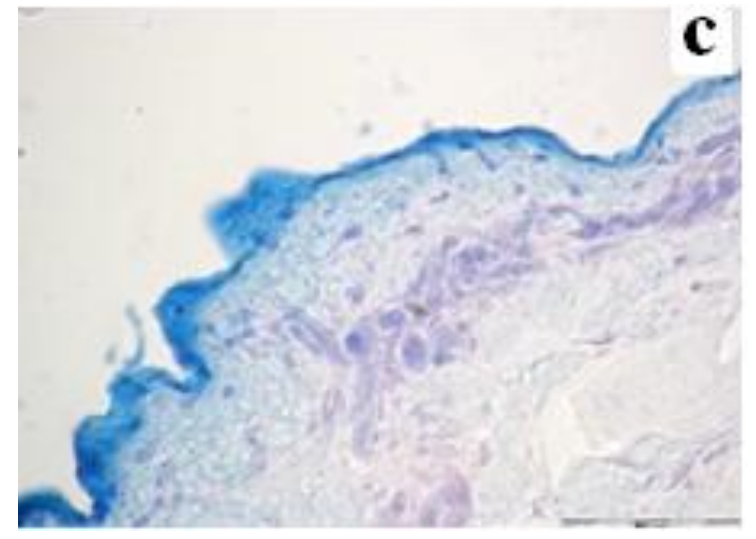

c: pig skin stimulated with 25 volts

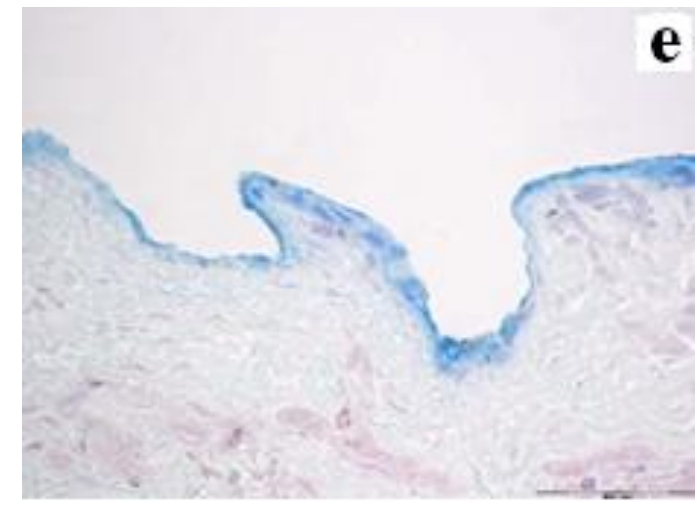

e: pig skin stimulated with 100 volts
monitored(Patricia $G$ et al.1998). The decrease in $\mathrm{MB}$ concentration in the skin with increasing voltage could be contributed to the structure and characteristic differences exist between low and high voltage electroporation(Seiji T et al. 2005).

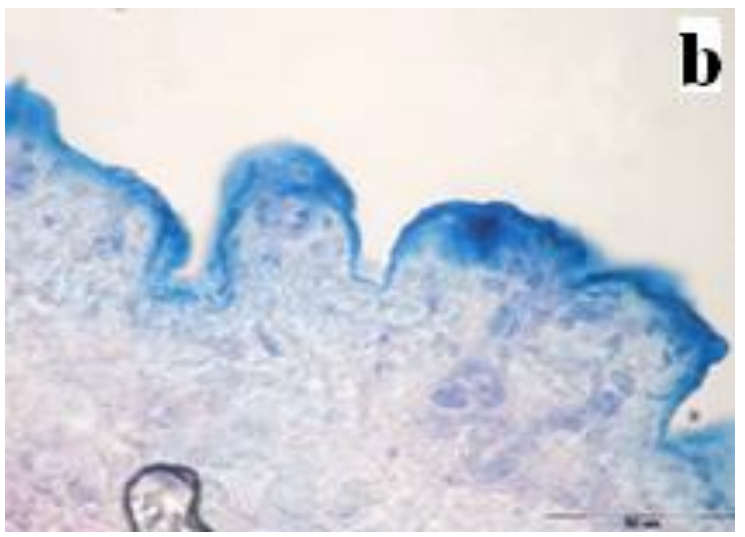

b: pig skin stimulated with 12.5 volts

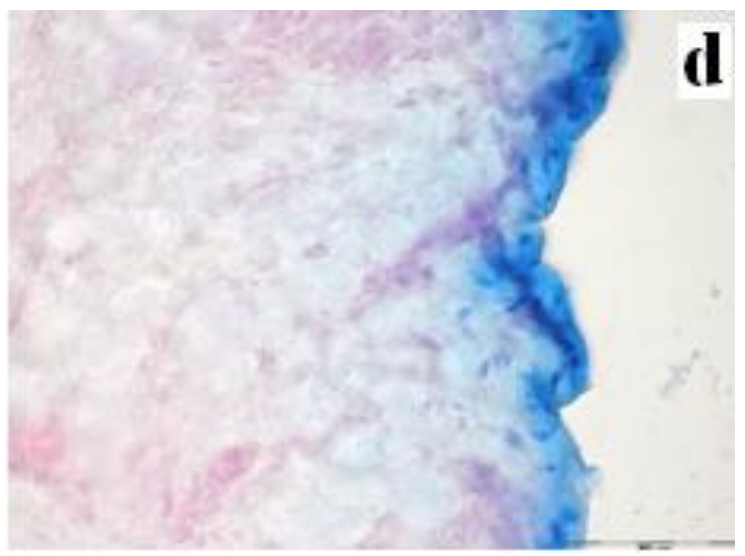

d: pig skin stimulated with 50 volts

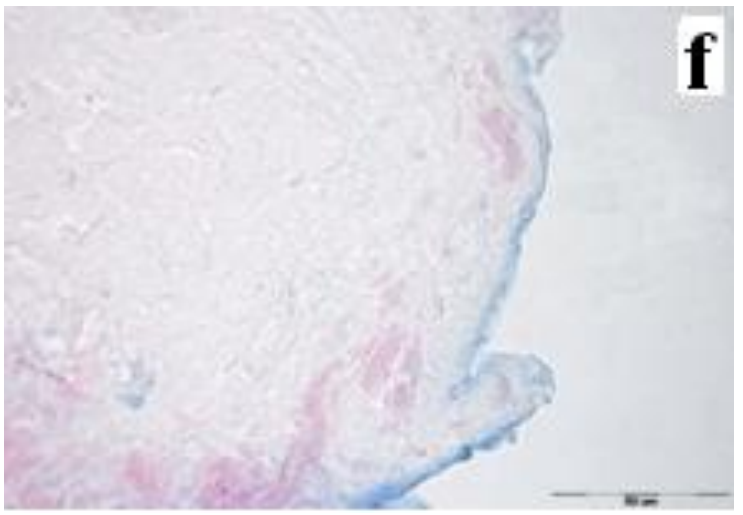

f: pig skin stimulated with 200 volts

Figure 6. Frozen section micrograph of pig skin stimulated with (6.5-200) volts showing the MB depth after stimulation $(X=400)$. 


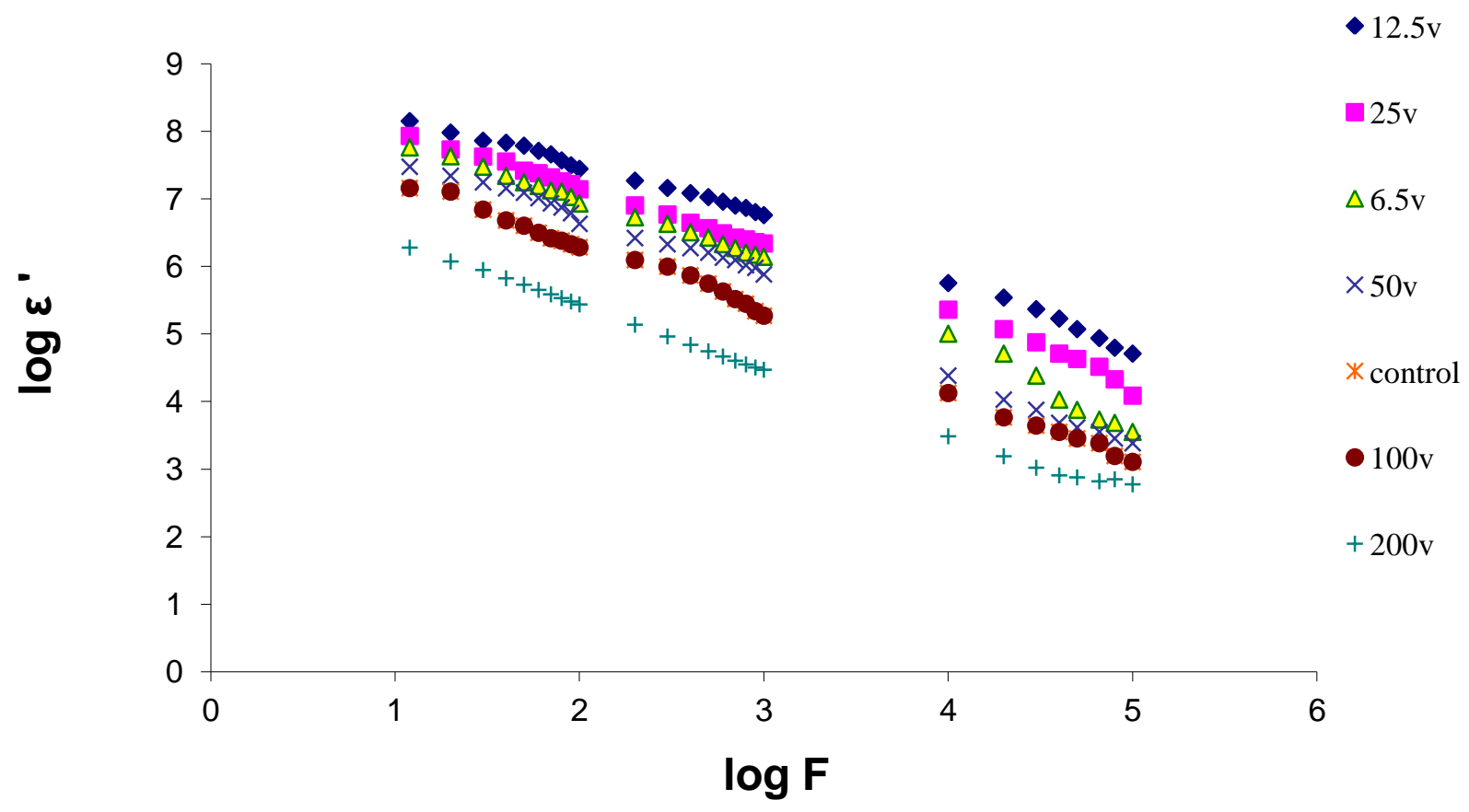

Figure 7. Thevariation of log pemittivity as function of log frequency for skin after stimulation.

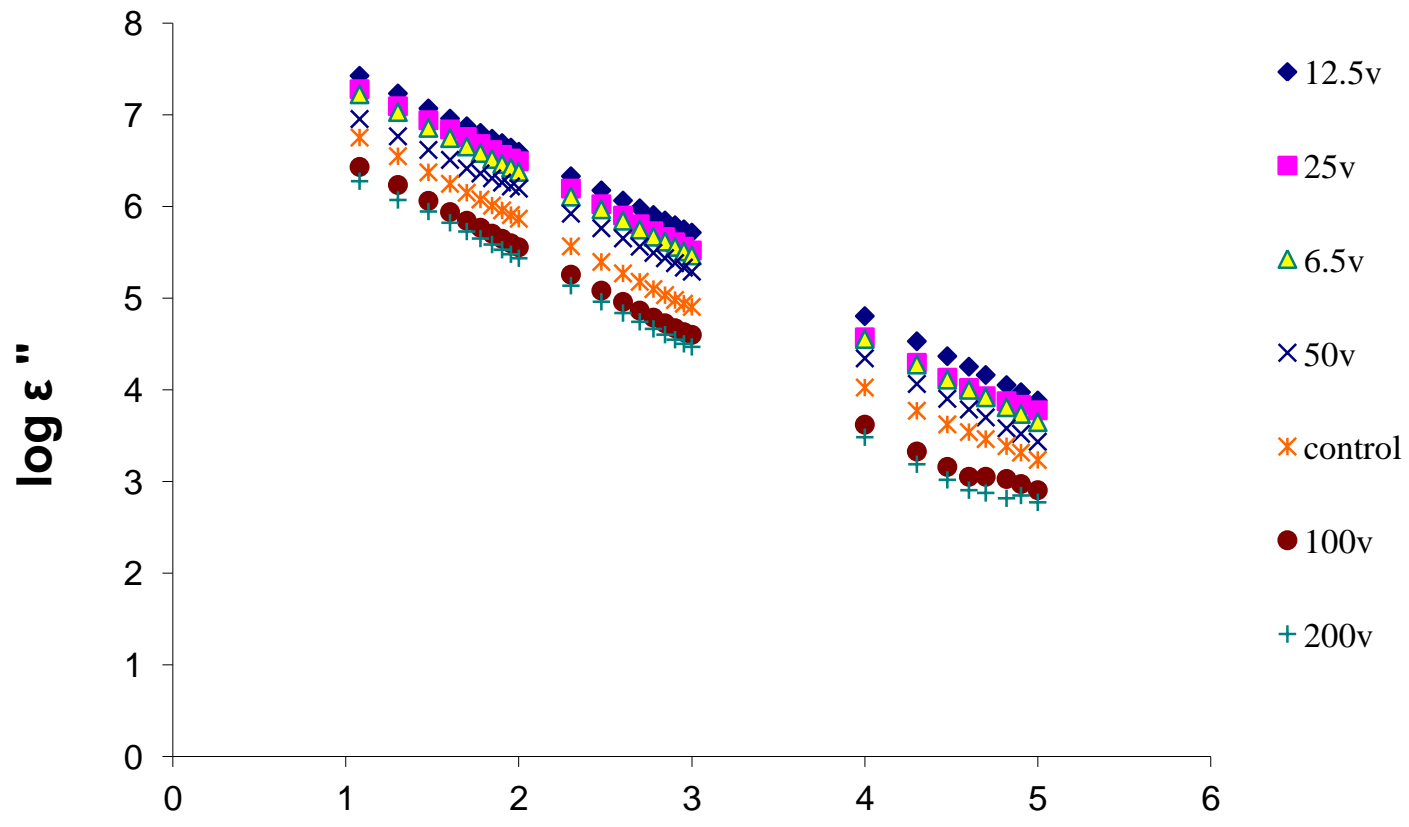

$\log f$

Figure 8. The variation of log dielectric loss as a function of log frequency for normal and stimulating skin. 


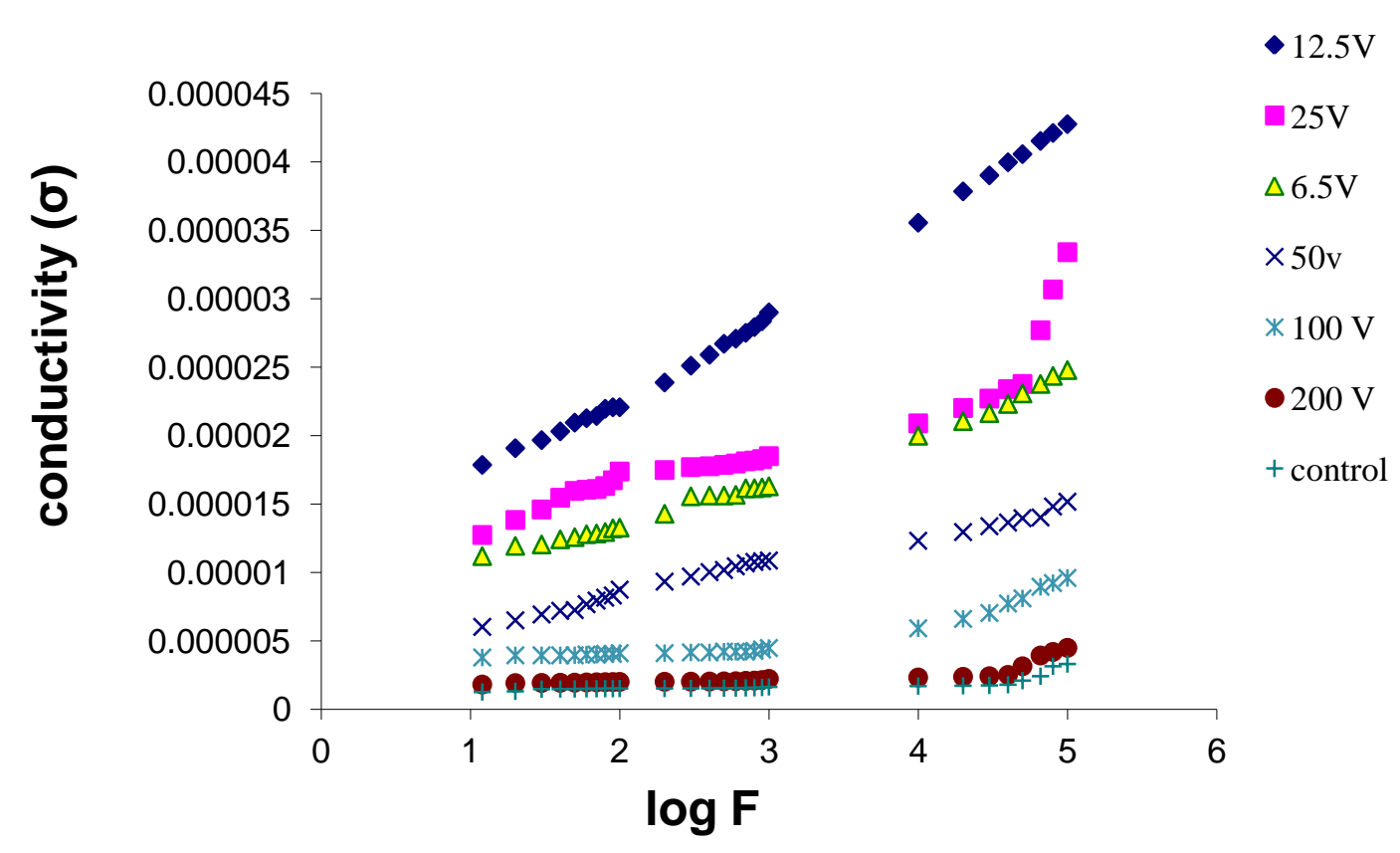

Figure 9. The variation of conductivity as a function of log frequency for skin after stimulation.

There is a high correlation coefficient between resistance and percentage of absorbed MB, $\left(\mathrm{R}^{2}=0.85\right)$. Revere(Riviere J1986), reported that, skin permeability of drug is greatly influenced by skin permselectivity. Since electroporation pretreatment damages the barrier function of the SC but does not strip the layer off.

Voltage, pulse length (pulse duration), number of pulses, and physicochemicalproperties of chemicals such as MB are among the factors affecting chemical or drug permeation in electroporation. Two different types of electrical pulsing protocols have been used: exponentially decaying pulses (ED) and square wave pulses (SW). A studyperformed by Vanbever et al. indicated that the intermittent application of short ( $\sim 1 \mathrm{~ms})$ high-voltage ( 100 across skin) ED pulses and a few applications of long (=100 ms) medium-voltage ( $>30 \mathrm{~V}$ across skin) ED pulses had similar alterations and recovery processes of skin electrical resistance, while long pulses of medium-voltageappeared to be more efficient in transporting sulforhodamine $(\mathrm{Mwt}=607)$ across skin. For the same total transported charge, long pulses induced faster and greatermolecular transport across skin than short pulses (Vanbever $R$ et al.1996).

Pulse length could be the second most important parameter next to the voltage determining electroporativein drug penetration. In Sharma etals. Study, $20 \mathrm{~V}$ pulses were used with lengths of 10, 20,30, and $40 \mathrm{~ms}$ to enhance the in vitro delivery of terazosin hydrochloride through hairless rat skin(Sharma A et al. 2000)

Resistance of skin measurements shown in figure. 4. reflect permeation of SC implying the creation or enlargement of SC opening through which electrolyte flow. The creation and/or the enlargement of aqueous pathways during electroporation has been proposed and observed in many studies. In an X-ray scattering analysis, disordering of lipid lamellar stacking and of the lipid lateral packing was observed after application of long electroporation pulses(Jadoul $A$ et al.1997). Freeze-fracture electron microscopy showed that high voltage pulses induced a general perturbation of the intercellular lipid materials. A light microscopy study found increased detachment in the SC cell layers with increasing electroporation voltages from 100 to 
300 V(Jadoul Aet al.1998). As resistance drop, voltage is redistributed across SC and the other layers, reducing the voltage gradient across the SC and reducing the probability that additional poration will occur(Patricia Get al.1998).

Electroporation produces tiny pores in the skin barrier and this decrease skin resistance(Vanbever $\mathrm{R}$ et al.1997) to confirm these results; we measured the dielectric properties of the pig skin after each stimulation; figures (7-9). The dielectric properties of the skin are largely determined by the SC which has thickness $15 \mu \mathrm{m}$ and consists of dead cells(Gabrial S t al.1996). By measuring the dielectric properties of pig skin in the frequency range $10 \mathrm{~Hz}-100 \mathrm{KHz}$, it can be noted that skin possesses a relatively weak $\alpha$-dispersion and this relative lack of significant dispersion is plausibly ascribed to the dead nature and low conductivity of the SC. It was suggested that the origin of these dispersion lay in SC and was associated with the relaxation of ions surrounding the corneal cells(Akimoto M et al. 2006). Since skin tissue after constitutes the interface between the biological and electronic parts of iontophoretic system, its dielectric properties are of some interest and importance.

\section{References}

1. Akimoto M, Kawahara M, Matsumoto $\mathrm{M}$, and Matsubayashi H (2006): Development of the pulsed direct current iontophoresis and its clinical application. Electromagnetics. 157162. doi: 10.2529/PIERS051008021701

2. Asbill C, El-kattan A, and Michniak B (2000): Enhancement of transdermal drug delivery: chemical and physical approaches crit. Rev. Ther. Drug carr. Syst. 17,621-658. doi: 10.1615/CritRevTherDrugCarrierSyst.v17.i6.20

3. Bhatia K, and Singh J (1999): Effect of linolenic acid / ethanol or limonene / ethanol and iontophoresis on the vitro percutaneous absorption of LHRH and ultrastructure of human epidermis , Int , J. pharm. 180:235250. doi: 10.1016/S0378-5173(99)00013-7
4. Burnette R, Hadgraft J, and Guy R (1989): Iontophoresis. In: Transdermal drug delivery. New York: Marcel Dekker. 247 - 291

5. Chien Y, and Banga A (1989):Iontophoretic (transdermal) delivery of drugs: overview of historical development, J.Pharma. SCi. 78, 353-354. doi: 10.1002/jps.2600780502

6. Chang S, and Riviere J (1991): Percutaneous absorption of parathion in porcine skin: Effects of dose, temperature, humidity and perfusate composition on absorptive flux. Fund. Appl. Toxicol.17, 494-504. doi: 10.1016/0272-0590(91)90200-N

7. Delgado - Charro M, and Guy R (2001): Transdermal iontophoresis for controlled drug delivery and non-invasive monitoring. STP pharma sci. 11,403-414

8. Diego M, Richard H. GuyR, Begoña DelgadoCharro (2001): Characterization of the iontophoreticpermselectivity properties of human and pig skin. 70, 213-217. doi: 10.1016/S0168-3659(00)00350-3

9. Flucker C, Sharpe P, Maslowski P, and Rowbothams D (2000):Iontophoretic application of methylene blue through porcine skin; influence ofpolarity. BrJ Ana. 84, 287291

10. Gabrial S, Lau W, and Gabrial C (1996): The dielectric properties of biological tissues:II. Measuemeants in the frequency range $10 \mathrm{~Hz}$ to $20 \mathrm{GHz}$. Phys. Med. Boil., vol.41. 41, 2251-2269. doi: $10.1088 / 0031$ 9155/41/11/002

11. Green P, lanagen M, Shroot B, and Guy R. (1993): Iontophoretic drug delivery. In: Walters KA, Hadgraf J, eds. Pharmaceutical skin penetration Enhancement. New York: Marcel Dekker. 311-333

12.Guy R (1996): Current status and future prospects of transdermal drug delivery.Pharm Res. 13, 1765-1769

13. Honggang S, Kevin L, Kendall D, David J, Miller I, and William H (2002): Improvement on conventional constant current DC iontophoresis: a study using constant conductance AC iontophoresis. J controlled Release. 82, 249-261. doi: 10.1016/S01683659(02)00107-4 
14. Jadoul A, Regnier V, Doucet J, Durand D, and Preat V (1997): X-ray scattering analysis of human stratum corneum treated by high voltage pulses, Pharm. Res. 14, 1275-1277

15. Jadoul A, Tanojo H, Preat V, Bouwstra J, Spies F, and Bodde H (1998): Electroperturbation of human stratum corneum fine structure by high voltage pulses: a freeze-fracture electron microscopy and differential thermal analysis study, J. Invest. Dermatol.Symp. Proc. 3, 153-158

16. Kalia, Y, Naik A, Garrison J, and Guy R (2004). iontophoretic drug delivery. Adv. 56,619-658. doi: 10.1016/j.addr.2003.10.026

17. Kassan D, Lynch A, and Stiller M. (1996): Physical enchancement of dermatologic drug delivery. Iontophoretsis and phonophoresis. J Am AcadDerm. 34, 657-666. doi: 10.1016/S0190-9622(96)80069-7

18. Khoo P, Michalak K, Yates P, Megson S (2006): Day R, and Wood D. Iontophoresis of antibiotics into segmental allograftsinin revision arthroplasty for infection . Br J Bone Joint Surg. 88,1430-1437. doi: 10.1302/0301620X.88B11.18335

19. Kirste J, Russell D, Cox B, David I, Vernon G, and Stanley B (2002): In Vitro Photodynamic Activity of a Series of Methylene Blue Analogues. Photochem and Photobiol; 75: 392-397. doi: 10.1562/00318655(2002)0750392IVPAOA2.0.CO2

20. Mitragori S, Blankschtein D, and Langer R (1995): Ultrasound-mediated transdermal protein delivery. Science. 269,850-853. doi: $\underline{10.1126 / \text { science. } 7638603}$
21. Monteiro-Riviere N (1986): Ultrastructural evaluation of the porcine integument. In: Swine in Biomedical Research (Tumbleson ME, Ed), Plenum Press, New York. 641-655

22. Narasimha M, Wiskirchen D, and Bowers P. (2007):Iontophoretic drug delivery across human nail. J Pharm Sci. 96, 305-311. doi: 10.1002/jps.20757

23. Patricia G, Stephen A, Sek W, and Allan R (1998): Apulsed electric field enhances cutaneous delivery of methylene blue in excised full- thickness porcine skin. J Invest Dermatol. 111, 457-463

24. Pearse A (1972) Histochemistry: Theoretical and Applied. Baltimore, Williams \& Wilkins. 1138-1139

25. Pradas R, Koul V, Anand S, and Khar (2007): Effect of $\mathrm{DC} / \mathrm{mDCiontophoresis} \mathrm{and} \mathrm{terpenes}$ on transdermal permeation of methotrexate: In vitro study. 333, 70- 78

26. Pradas R, Koul V, Anand S, and Khar (2006): Effect of DC/mDCiontophoresis and terpenes on transdermal permeation of methotrexate: In vitro study. 9, 58-62. doi: 10.1016/j.ijpharm.2006.09.058

27. Prausnitz M, Bose V, Langer R, and Weaver J (1993): Electroporation of mammalian skin: a mechanism to enhance transdermal drug delivery, Proc. Natl. Acad. SCi U.S.A. go. 10504-10508 